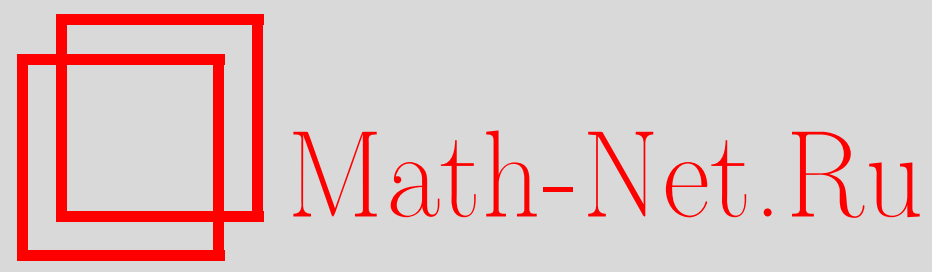

А. А. Локшин, О выпуклости поверхности собственных значений, Матем. заметки, 1997, том 61, выпуск 6, 946-949

DOI: https://doi.org/10.4213/mzm1580

Использование Общероссийского математического портала Math-Net.Ru подразумевает, что вы прочитали и согласны с пользовательским соглашением http://www. mathnet.ru/rus/agreement

Параметры загрузки:

IP : 35.174 .16 .151

26 апреля 2023 г., 13:03:58

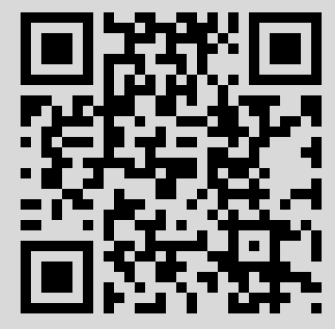




\section{О ВЫПУКЛОСТИ ПОВЕРХНОСТИ СОБСТВЕННЫХ ЗНАЧЕНИЙ}

\section{А. А. Локшин}

В заметке устанавливается результат, принадлежащий к классу теорем типа Гельмана-Фейнмана (см. [1]). Именно, показывается, что для действующего в вещественном гильбертовом пространстве $\mathscr{H}$ полуограниченного снизу самосопряженного оператоpa, линейно зависящего от $n$ вещественных числовых параметров и такого, что нижняя точка его спектра представляет собой изолированное невырожденное собственное значение, это собственное значение оказьвается вьпуклой вверх функцией упомянутых параметров. В случае $\mathscr{H}=L_{2}\left(\mathbb{R}^{k}\right)$ сформулированньй результат обобщается на один специальный класс операторов, нелинейно зависящих от числовых параметров.

1. Пусть $\mathscr{H}$ - вещественное гильбертово пространство.

ТЕОРема 1. Пусть $H_{0}$ - самосопряженный полуограниченный снизу оператор с областью определения $D\left(H_{0}\right) \subset \mathscr{H} ; V_{i}, i=1, \ldots, n,-$ ограниченные симметрические операторы, определенные на всем भ̈. Рассмотрим оператор

$$
H=H_{0}+a_{1} V_{1}+\cdots+a_{n} V_{n}, \quad a_{i} \in \mathbb{R}^{1}, \quad i=1, \ldots, n
$$

Предположим, что при каждом $a=\left(a_{1}, \ldots, a_{n}\right)$ из достаточно малого шара

$$
\left(a_{1}^{2}+\cdots+a_{n}^{2}\right)^{1 / 2}<\varepsilon, \quad \varepsilon>0
$$

нижняя грань спектра полуограниченного снизу самосопряжснного оператора $H=H($ a) представляет собой изолированное невыроэсденое собственное значение $E=E(a)$ (не ограничивая общности, считаем $E(a)$ и соответствующий нормированный собственный вектор $\psi($ (а) вещественно-аналитическими в иаре (1)). Тогда в иаре (1) функция E(a) выпукла вверх.

ЛЕмма. В условиях теоремы 1

$$
\frac{\partial \psi(a)}{\partial a_{i}} \in D\left(H_{0}\right)
$$

u, кроме того,

$$
\frac{\partial}{\partial a_{i}} H_{0} \psi(a)=H_{0} \frac{\partial \psi(a)}{\partial a_{i}}
$$

Работа выполнена при финансовой поддержке Российского фонда фундаментальных исследований, грант № 95-01-01171а. 
ДоКАЗАТЕЛЬСТво. Рассмотрим соотношение

$$
\frac{\psi\left(a+\Delta a_{i}\right)-\psi(a)}{\Delta a_{i}} \in D\left(H_{0}\right),
$$

где $a+\Delta a_{i}=\left(a_{1}, \ldots, a_{i}+\Delta a_{i}, \ldots, a_{n}\right)$. В силу вещественной аналитичности $\psi(a)$ существует сильньй предел этого отношения при $\Delta a_{i} \rightarrow 0$, которьй мы и обозначим через $\partial \psi / \partial a_{i}$. Далее имеем

$$
\begin{aligned}
H_{0} & \frac{\psi\left(a+\Delta a_{i}\right)-\psi(a)}{\Delta a_{i}} \\
= & \frac{1}{\Delta a_{i}}\left\{H\left(a+\Delta a_{i}\right) \psi\left(a+\Delta a_{i}\right)-\left[a_{1} V_{1}+\cdots+\left(a_{i}+\Delta a_{i}\right) V_{i}+\cdots+a_{n} V_{n}\right] \psi\left(a+\Delta a_{i}\right)\right. \\
& \left.-H(a) \psi(a)+\left[a_{1} V_{1}+\cdots+a_{i} V_{i}+\cdots+a_{n} V_{n}\right] \psi(a)\right\} \\
= & \frac{1}{\Delta a_{i}}\left\{E\left(a+\Delta a_{i}\right) \psi\left(a+\Delta a_{i}\right)-E(a) \psi(a)-a_{1} V_{1}\left[\psi\left(a+\Delta a_{i}\right)-\psi(a)\right]-\cdots\right. \\
& \left.-\left[\left(a_{i}+\Delta a_{i}\right) V_{i} \psi\left(a+\Delta a_{i}\right)-a_{i} V_{i} \psi(a)\right]-\cdots-a_{n} V_{n}\left[\psi\left(a+\Delta a_{i}\right)-\psi(a)\right]\right\},
\end{aligned}
$$

откуда, очевидно, следует существование сильного предела при $\Delta a_{i} \rightarrow 0$ величины, стоящей в левой части последнего равенства. Теперь утверждения (2) и (3) леммы следуют из замкнутости самосопряженного оператора $H_{0}$.

СлЕДСТВИЕ. В условиях теоремы 1

$$
(H(a) \psi(a))_{a_{i}}^{\prime}=V_{i} \psi+H \psi_{a_{i}}^{\prime}
$$

ДоКАЗАТЕЛЬСТво ТЕОРЕмЫ 1. Как это обьчно делается при доказательстве теорем типа Гельмана-Фейнмана (см., например, [2]), будем исходить из соотношения

$$
\langle\psi(a),(H(a)-E(a)) \psi(a)\rangle=0, \quad a=\left(a_{1}, \ldots, a_{n}\right)
$$

$(\langle\cdot, \cdot\rangle$ означает скалярное произведение в $\mathscr{H})$. Дифференцируя (5) по $a_{i}$, с учетом (4) и самосопряженности оператора $H(a)$ получаем $\left\langle\psi,\left(V_{i}-E_{a_{i}}^{\prime}\right) \psi\right\rangle=0$. Дифференцируя теперь это соотношение по $a_{j}$, с учетом самосопряженности $V_{i}$ имеем

$$
2\left\langle\psi_{a_{j}}^{\prime},\left(V_{i}-E_{a_{i}}^{\prime}\right) \psi\right\rangle-E_{a_{i} a_{j}}^{\prime \prime}\langle\psi, \psi\rangle=0
$$

Далее, дифференцируя по $a_{j}$ равенство $(H-E) \psi=0$, с учетом (4) имеем

$$
(H-E) \psi_{a_{j}}^{\prime}+\left(V_{j}-E_{a_{j}}^{\prime}\right) \psi=0
$$

откуда, в частности, следует, что

$$
\left(V_{j}-E_{a_{j}}^{\prime}\right) \psi \in\{\operatorname{Ker}(H-E)\}^{\perp}
$$

где через $\{\operatorname{Ker}(H-E)\}^{\perp}$ обозначено ортогональное дополнение к $\operatorname{Ker}(H-E)$ в $\mathscr{H}$. Из соотношений $(7),(8)$ имеем

$$
\psi_{a_{j}}^{\prime}=-(H-E)^{-1}\left(V_{j}-E_{a_{j}}^{\prime}\right) \psi
$$


Здесь $(H-E)^{-1}$ понимается как оператор, действующий на $\{\operatorname{Ker}(H-E)\}^{\perp}$.

Подставим теперь (9) в (6). Тогда, учитьвая нормированность вектора $\psi$, получим

$$
-\frac{\partial^{2} E}{\partial a_{i} \partial a_{j}}=2\left\langle(H-E)^{-1}\left(V_{j}-E_{a_{j}}^{\prime}\right) \psi,\left(V_{i}-E_{a_{i}}^{\prime}\right) \psi\right\rangle .
$$

Нетрудно видеть, что ограниченный симметрический оператор $(H-E)^{-1}$ положительно определен $\left(\right.$ на $\left.\{\operatorname{Ker}(H-E)\}^{\perp}\right)$. Следовательно, на $\{\operatorname{Ker}(H-E)\}^{\perp}$ определен и квадратный корень из него, который мы обозначим через $(H-E)^{-1 / 2}$. Положим

$$
\xi_{l}=\sqrt{2}(H-E)^{-1 / 2}\left(V_{l}-E_{a_{l}}^{\prime}\right) \psi, \quad l=1, \ldots, n .
$$

Тогда (10), очевидно, перепишется следуюшим образом:

$$
-\frac{\partial^{2} E}{\partial a_{i} \partial a_{j}}=\left\langle\xi_{j}, \xi_{i}\right\rangle, \quad i, j=1, \ldots, n .
$$

Однако, как хорошо известно, матрица Грама $\left\|\left\langle\xi_{j}, \xi_{i}\right\rangle\right\|$ неотрицательно определена. Следовательно, в силу (11) в шаре (1) неотрицательно определен гессиан функции $-E(a)$, откуда следует выпуклость вверх функции $E(a)$ в упомянутом шаре. Теорема доказана.

2. Перейдем теперь к случаю, когда вещественное гильбертово пространство $\mathscr{H}=$ $L_{2}\left(\mathbb{R}^{k}\right)$. Всюду ниже считаем $V_{s}, s=1, \ldots, m$, операторами умножения на функции от $x=\left(x_{1}, \ldots, x_{k}\right)$. Теперь покажем, что можно определить довольно широкое семейство "потенщиалов" $V_{s}$, нелинейным образом зависящих от параметров $a_{1} \geqslant 0, \ldots, a_{n} \geqslant 0$ и таких, что справедливо утверждение, аналогичное утверждению теоремы 1.

Итак, пусть

$$
\begin{gathered}
V_{s}=V_{s}\left(f_{0 s}(x)+a_{1} f_{1 s}(x)+\cdots+a_{n} f_{n s}(x)\right), \\
f_{i s}(x) \geqslant 0, \quad f_{i s}(x) \in C^{2}\left(\mathbb{R}^{k}\right), \quad V_{s}(y) \in C^{2}[-1, \infty),
\end{gathered}
$$

причем

$$
\frac{d^{2} V_{s}(y)}{d y^{2}} \leqslant 0 \quad \text { при } \quad y \geqslant-1 .
$$

Рассмотрим оператор

$$
H(a)=H_{0}+V_{1}+\cdots+V_{m},
$$

где $H_{0}$ - некоторый полуограниченньй снизу самосопряженный оператор в $L_{2}\left(\mathbb{R}^{k}\right)$.

ТЕорема 2. Пусть выполнены ограничения (12) на функции $f_{i s} u V_{s}$. Предположим, далее, что в некоторой области $\Omega$ изменения неотричательных параметров $a_{1}, \ldots, a_{n}$ оператор (13) самосопряжен, причем нижняя грань его спектра представляет собой изолированное невырожденное собственное значение $E=E(a), a=\left(a_{1}, \ldots, a_{n}\right)$. Кроме того, предположим, что $E(a)$ в $\Omega$ является дважды непрерывно дифференцируемой функиией от $а, \quad$ a соответствующий нормированный собственный вектор $\psi=\psi(a)$ дважды сильно непрерывно дифференцируем по а. Тогда при $a \in \Omega$ функиия $E(a)$ выпукла вверх. 
ДоКАЗАТЕЛЬСТВО. Прежде всего, заметим, что утверждения леммы сохраняются и в условиях теоремы 2 , а соотношение (4) приобретает вид

$$
(H(a) \psi(a))_{a_{i}}^{\prime}=H_{a_{i}}^{\prime} \psi+H \psi_{a_{i}}^{\prime}
$$

Поэтому, дифференцируя (5) по $a_{i}$, с учетом самосопряженности оператора $H$ имеем

$$
\left\langle\psi,\left(H_{a_{i}}^{\prime}-E_{a_{i}}^{\prime}\right) \psi\right\rangle=0
$$

Дифференцируя это соотношение по $a_{j}$ (что, очевидно, возможно в условиях теоремы) и используя симметричность ограниченного оператора $H_{a_{i}}^{\prime}$, имеем

$$
2\left\langle\psi_{a_{j}}^{\prime},\left(H_{a_{i}}^{\prime}-E_{a_{i}}^{\prime}\right) \psi\right\rangle+\left\langle\psi,\left(H_{a_{i} a_{j}}^{\prime \prime}-E_{a_{i} a_{j}}^{\prime \prime}\right) \psi\right\rangle=0
$$

Аналогично (9) с помощью (14) имеем

$$
\psi_{a_{j}}^{\prime}=-(H-E)^{-1}\left(H_{a_{j}}^{\prime}-E_{a_{j}}^{\prime}\right) \psi
$$

Подставляя (16) в равенство $(15)$, получаем

$$
\begin{aligned}
-\frac{\partial^{2} E}{\partial a_{i} \partial a_{j}}= & 2\left\langle(H-E)^{-1 / 2}\left(H_{a_{j}}^{\prime}-E_{a_{j}}^{\prime}\right) \psi,(H-E)^{-1 / 2}\left(H_{a_{i}}^{\prime}-E_{a_{i}}^{\prime}\right) \psi\right\rangle \\
& +\sum_{s=1}^{m}\left\langle\psi,-V_{s}^{\prime \prime} f_{i s} f_{j s} \psi\right\rangle, \quad i, j=1, \ldots, n
\end{aligned}
$$

Положим теперь

$$
\xi_{l}=\sqrt{2}(H-E)^{-1 / 2}\left(H_{a_{l}}^{\prime}-E_{a_{l}}^{\prime}\right) \psi, \quad \eta_{l s}=f_{l s} \psi \sqrt{-V_{s}^{\prime \prime}}, \quad l=1, \ldots, n, \quad s=1, \ldots, m .
$$

Тогда (17), очевидно, перепишется следуюшим образом:

$$
-\frac{\partial^{2} E}{\partial a_{i} \partial a_{j}}=\left\langle\xi_{i}, \xi_{j}\right\rangle+\sum_{s=1}^{m}\left\langle\eta_{i s}, \eta_{j s}\right\rangle, \quad i, j=1, \ldots, n .
$$

Итак, гессиан функции $-E(a)$ представим в виде суммы $n+1$ матриц Грама и, тем самым, является положительно определенной матрицей. Теорема доказана.

\section{СПИСОК ЦИТИРОВАННОЙ ЛИТЕРАТУРЫ}

1. Цюлике Л. Квантовая химия. Т. 1. М.: Мир, 1976. 2. Эпштейн С. Вариационньй метод в квантовой химии. М.: Мир, 1977. 